\title{
Mated Flight Control Issues for Space Exploration Systems
}

\author{
Kyong B. Lim ${ }^{*}$, F. Landis Markley ${ }^{\dagger}$, and Mark S. Whorton ${ }^{\ddagger}$ \\ National Aeronautics \& Space Administration
}

\begin{abstract}
Several unique issues related to mated flight control have been broadly identified. These issues include redundancies in subsystems, controllability, command and control authority distribution, information flow across elements, and changes and variability in system characteristics due to variable mated configurations during operations. Architectural options for mated flight control are discussed in the context of evolving space systems.
\end{abstract}

\subsection{INTRODUCTION}

A successful execution of a space exploration mission (see for example [1]-[2]) will require extensive utilization of mated flight control (MFC) capability and operations infrastructure whereby two or more vehicles can be operated together as a single flight unit. This paper identifies some key issues unique to MFC and examines available options for a subset of these with a goal of defining necessary needed technologies concerning MFC. In addition, identifying key issues and options can help to clarify necessary requirements for an evolving space system early on for logistical reasons.

Past and current mated configurations were investigated to gain insight into key issues that are relevant to MFC such as International Space Station (ISS) - Orbital Space Plane (OSP) [3,4], Command Service Module (CSM) - Lunar Module (LM) Apollo [5,6], and ISS - Visiting Vehicle (VV) [7]. This helped to illuminate and confirm a few issues relevant to mated flight control operations. For example, on the issue of locus of control, i.e. the designation of what entity (ground, element automation, or crew) is authorized to issue commands to a spacecraft, for operations today, the locus of control typically resides at the spacecraft. When two vehicles rendezvous (such as Shuttle docking at the ISS or Shuttle retrieving a payload), one spacecraft is designated as the controlling entity while the other spacecraft is the passive target.

To support exploration missions, inter-element command and control autonomy and automation will be needed that requires shifting the locus of control, depending upon the mission phase and configuration of vehicles. Historically, ground controllers monitored telemetry and issued commands by voice to the crew (such as crucial navigation and systems management commands) when communication was available. In a few cases, such as control of Apollo antenna direction, the ground directly controlled spacecraft systems, to remove that distraction from the crew's workload. For the Space Shuttle, control is accomplished by flight software or the crew. Crew command sequences are preplanned on the ground as part of crew procedures. The ground also uplinks data that can affect command and control (e.g., state vector updates) but does not uplink direct control commands. Analysis indicates that manned operations have become increasingly more reliant on remote command and control from ground. For the International Space Station, most commanding is done from the ground. Like the Shuttle, command sequences are preplanned as procedures. Unlike the Shuttle, however, most human control for ISS is

\footnotetext{
* Senior Research Engineer, Dynamic Systems \& Control Branch, Langley Research Center.

${ }^{\dagger}$ Senior Research Scientist, Integrated GNC Branch, Goddard Space Flight Center.

‡ Branch Chief, Guidance, Navigation, and Mission Analysis, Marshall Space Flight Center.
} 
done by the ground. Original plans for ISS included onboard automated control for many vehicle systems; however this proved cost prohibitive. Hence, the ground flight controllers currently perform many of these functions, retreating from both autonomy and automation. This history of station automation has interesting implications for mated flight control. For Russian spacecraft, a review of historical literature indicates that Vostok, Voskhod, Soyuz, Progress, and Buran were all largely automated, with minimal crew command and control; taking the opposite of the US approach during the same eras. It has been observed, however, that numerous failures of automatic systems and unsuccessful manual dockings in the absence of onboard guidance computers significantly slowed the Soviet program.

This study identifies and examines issues primarily from the Guidance, Navigation, and Control (GN\&C) viewpoint for a class of mated vehicles. Possible options to address some of these issues are then discussed. There are other important MFC/GN\&C related aspects of mated flight control operations that this paper will not address such as rendezvous and docking, command and control interoperability, autonomy and automation, and software system reuse, interoperability, and compatibility.

\subsection{ISSUES UNIQUELY RELEVANT TO MFC}

The exploration missions require the capability for two or several vehicles to be operated together as a single flight unit. This requires GN\&C and command and data handling (C\&DH), and command and control intercommunication (CCI) between mated elements. The requirements include command and control authority distribution options, information flow across mated elements, changes in vehicle static and dynamic characteristics, verification and validation $(\mathrm{V} \& \mathrm{~V})$ of mated vehicle dynamics, GN\&C configuration changes, interface mechanism variability, articulation of vehicle components, mass redistribution, and adaptation to multiple flight configurations and operating modes. Each element will have its own GN\&C subsystem, capable of executing GN\&C functions and some degree of built-in redundancy for that element, but not necessarily adequate for the mated vehicles. Exploration matedelement command and control will require shifting the locus of control among mated elements, depending upon the mission configuration and phase. MFC command and control requirements for a spacecraft element may require over-design (to accommodate larger mass properties envelope and structural characteristics). Each element will have independent actuator sets capable of controlling that element, but perhaps not adequate for the mated vehicles. A feasible actuator set must provide sufficient control authority for MFC to satisfy performance requirements for all control modes, mated configurations, and planned missions and fit within allocated budgets including mass, power, and volume. Necessary information (states, data, command signals, etc) must flow across the inter-element interfaces for MFC operations.

In this study, we assume that the flight control functional and performance requirements for a single spacecraft are understood and well known. The focus in this study is then to identify and examine major issues related to flight control (more generally GN\&C aspects) that are expected to be unique as a consequence of in-space mating of these individually well known and understood elements. The following list represents the unique issues related to MFC identified in this study:

- Redundancies in Subsystems/Components

o Plurality of GN\&C components

o Mission success, Crew safety

- Controllability

o Sufficient control authority

o Actuator hardware (type, sizing), configuration, vehicle dynamics, etc

- Command \& Control (C2) authority distribution 
o Assignment of C2 authority, to meet common goals

- Information flow across mated elements

o Minimal information set

o Abstraction level of information

o Specification and documentation of interface requirements

- Changes in static and dynamic characteristics

o Simulation modeling required for V\&V of GN\&C functionality

o Configuration changes, interface mechanism variability, articulation, mass redistribution

o MFC robustness to combined uncertainties \& disturbances

o V\&V of mated vehicle dynamics

- Flight Controller adaptation to varying flight configurations and operating modes

The above list is not intended to be an all encompassing and exhaustive list of issues that are unique to MFC, rather, it is intended to be a suitable springboard for further study. In addition, each of the issues identified above may be closely related and therefore cannot be addressed independently, for example, MFC performance robustness issues are implicitly related to vehicle controllability and issues related to variability and uncertainties in the static and dynamic characteristics of the mated vehicle.

\subsection{EVOLVING STRUCTURE}

\subsection{MFC Architecture}

In the previous section, we discussed main issues that are considered unique to MFC, in a generic and broad perspective. In this section, we discuss in more detail a subset of these identified issues in the context of an evolving spacecraft system that changes its configuration during operation.

Consider three specific MFC architectures distinguished by their differences in which element controls the mated stack using what resources, as shown in Table 1. The analysis considers mostly 2-element mated configurations. Although there are many influencing factors in MFC architecture selection such as: GN\&C requirements, failure mode characteristics, redundancy requirements, data interface requirements and complexity, $\mathrm{V} \& \mathrm{~V}$ feasibility and cost, structural flexibility of mated system, control technology maturation (e.g., reconfigurable, intelligent control, etc.), function of elements and mated systems, we focus only on the dependence of MFC architecture on GN\&C functional and performance capability, and in particular on Stability and Control (S\&C) aspects.

\subsection{Redundancies}

Each element will have its own GN\&C subsystem, capable of executing GN\&C functions and with some degree of built-in redundancy that is adequate for that element, but not necessarily for the mated vehicle. The issue is how to exploit the multiplicity of GN\&C components over all mated elements for MFC to (1) build-in redundancies against failures in these mated vehicles, to ensure Mission Success and Crew Safety, and (2) construct viable GN\&C subsystems for multiple mated vehicle configurations. The focus is on GN\&C component redundancy aspects for MFC and not Life support, Communication, Command \& Control, etc., aspects of the mated vehicle. 
Table 1 MFC Architectural Options

\begin{tabular}{|c|c|c|c|}
\hline & $\begin{array}{l}\quad \text { Architecture 1: } \\
\text { Augmented Vehicle } \\
\text { Single element assumes complete } \\
\text { GNC responsibility for mated } \\
\text { vehicle using only its resources. }\end{array}$ & $\begin{array}{l}\text { Architecture 2: } \\
\text { Centralized Control } \\
\text { Single element assumes complete } \\
\text { GNC responsibility for mated vehicle } \\
\text { using resources from all elements. }\end{array}$ & $\begin{array}{l}\text { Architecture 3: } \\
\text { Decentralized Control } \\
\text { All elements help maintain GNC } \\
\text { responsibility for the mated vehicle. }\end{array}$ \\
\hline Controllability & Low/Moderate, may require over-design & High & High \\
\hline $\begin{array}{l}\text { Mating Transient } \\
\text { Risks }\end{array}$ & $\begin{array}{l}\text { Low, since the same control } \\
\text { configuration will remain operational } \\
\text { before and after the mating. }\end{array}$ & $\begin{array}{l}\text { Moderate risk, since centralized } \\
\text { controller with a different configuration } \\
\text { takes over control from element } \\
\text { controllers. }\end{array}$ & $\begin{array}{l}\text { Moderate risk, due to the complexity in } \\
\text { the transient behavior of highly coupled } \\
\text { multiple adaptive controllers }\end{array}$ \\
\hline $\begin{array}{l}\text { Control } \\
\text { Reconfiguration \& } \\
\text { Adaptation } \\
\text { Requirement }\end{array}$ & $\begin{array}{l}\text { Controller reconfiguration is } \\
\text { unnecessary even during mating } \\
\text { transients. Simple gain scheduling } \\
\text { will likely be required. }\end{array}$ & $\begin{array}{l}\text { Controller reconfiguration will be } \\
\text { necessary during mating transient. An } \\
\text { adaptive capability will likely be needed } \\
\text { to gently phase in the new control } \\
\text { structure. }\end{array}$ & $\begin{array}{l}\text { Controller reconfiguration is unnecessary } \\
\text { during mating transients. Adaptive } \\
\text { capability in the form of a supervisory } \\
\text { control, beyond gain scheduling will } \\
\text { likely be required. }\end{array}$ \\
\hline $\begin{array}{c}\text { Interface } \\
\text { Requirements }\end{array}$ & $\begin{array}{l}\text { Low, no GNC data or commands } \\
\text { need to pass between elements. }\end{array}$ & $\begin{array}{l}\text { High, since GNC data must pass } \\
\text { between elements. May require data } \\
\text { transfer prior to mating for initialization. }\end{array}$ & $\begin{array}{l}\text { High, since GNC data must pass between } \\
\text { elements. May require data transfer prior } \\
\text { to mating for initialization. }\end{array}$ \\
\hline $\begin{array}{l}\text { Controller } \\
\text { Performance \& } \\
\text { Complexity }\end{array}$ & Low, Low & High, Moderate & $\begin{array}{l}\text { High, but increased risk for performance } \\
\text { variability and instability. High, since } \\
\text { decentralized controllers would likely } \\
\text { require a supervisory controller \& FDIR } \\
\text { method. }\end{array}$ \\
\hline Cost & Low & $\begin{array}{l}\text { High, need to integrate verify complex } \\
\text { interfaces. }\end{array}$ & $\begin{array}{l}\text { High, need to integrate verify complex } \\
\text { interfaces. }\end{array}$ \\
\hline Program Risk & Low & $\begin{array}{l}\text { Moderate, due to increase in system } \\
\text { complexity \& reconfigurability . Risks } \\
\text { from integration \& verification of } \\
\text { complex interfaces. }\end{array}$ & $\begin{array}{l}\text { High, due to technology advancements } \\
\text { needed. Risks from integration \& } \\
\text { verification of complex interfaces. }\end{array}$ \\
\hline
\end{tabular}

An option to manage redundancy that was used successfully during Apollo program, specifically for the mated configuration CSM-LM [3], is to allocate control functions with redundancy capability to individual elements. This is analogous to wrapping a sequence of single-loop controllers based on wellestablished classical control ideas to control a multivariable system, wherein each control function is allocated to a specific loop. As an important special case, we use one of two vehicles to control the mated stack, for example, Apollo's CSM controlling the CSM-LM stack. This special case corresponds to MFC Architecture 1, as described in Table 1.

As an illustration, Figure 1 shows how one can allocate functions to a particular mated configuration consisting of two elements, V1 and V2, where V1 and V2 denote vehicle 1 and vehicle 2, respectively. Notice that several options arise based on the particular choice of 4-DOF control function allocation, as compared to for example 6-DOF control function. Subsequently, requirements on both vehicles can be easily identified. For example, as a MFC requirement on V1, to allow all 4-DOF MFC options with V2, V1 must have 3-DOF rotational control and command of V1's translational jet. Obviously, the trade space can be populated along this line as necessary, given sufficient time and effort.

An alternate approach is to use all available elements for MFC and to satisfy redundancy requirements for the mated vehicle. This corresponds to MFC Architecture 2 and 3 in Table 1. The analogy is one of multivariable control approach as compared to classical single loop control. Instead of allocating specific functions (say Roll axis control), to specific components (say thrust RCS on a specific element), this alternate approach relies on a judiciously selected performance metric to optimally manage redundant components (computers, sensors, and actuators) for a common control objective. This approach has several advantages. One advantage is that it can provide enhanced performance especially when separately allocated functions are tightly coupled. Another advantage is the inherent flexibility in the approach if control reconfiguration is required. This approach can also save resources by eliminating the 
need to provide duplicate functions on the different elements. This approach also has some drawbacks. Reducing the risk and ensuring the performance of the mated system depends on more intensive information exchange between elements and a higher level of hardware and software complexity in the mated interface. These will likely lead to extra costs in the integration and verification of the more complex multiple vehicle interfaces. The tradeoff between these additional costs and the potential savings of eliminating duplication between individual elements must be carefully balanced. More comparisons are given in Table 1.

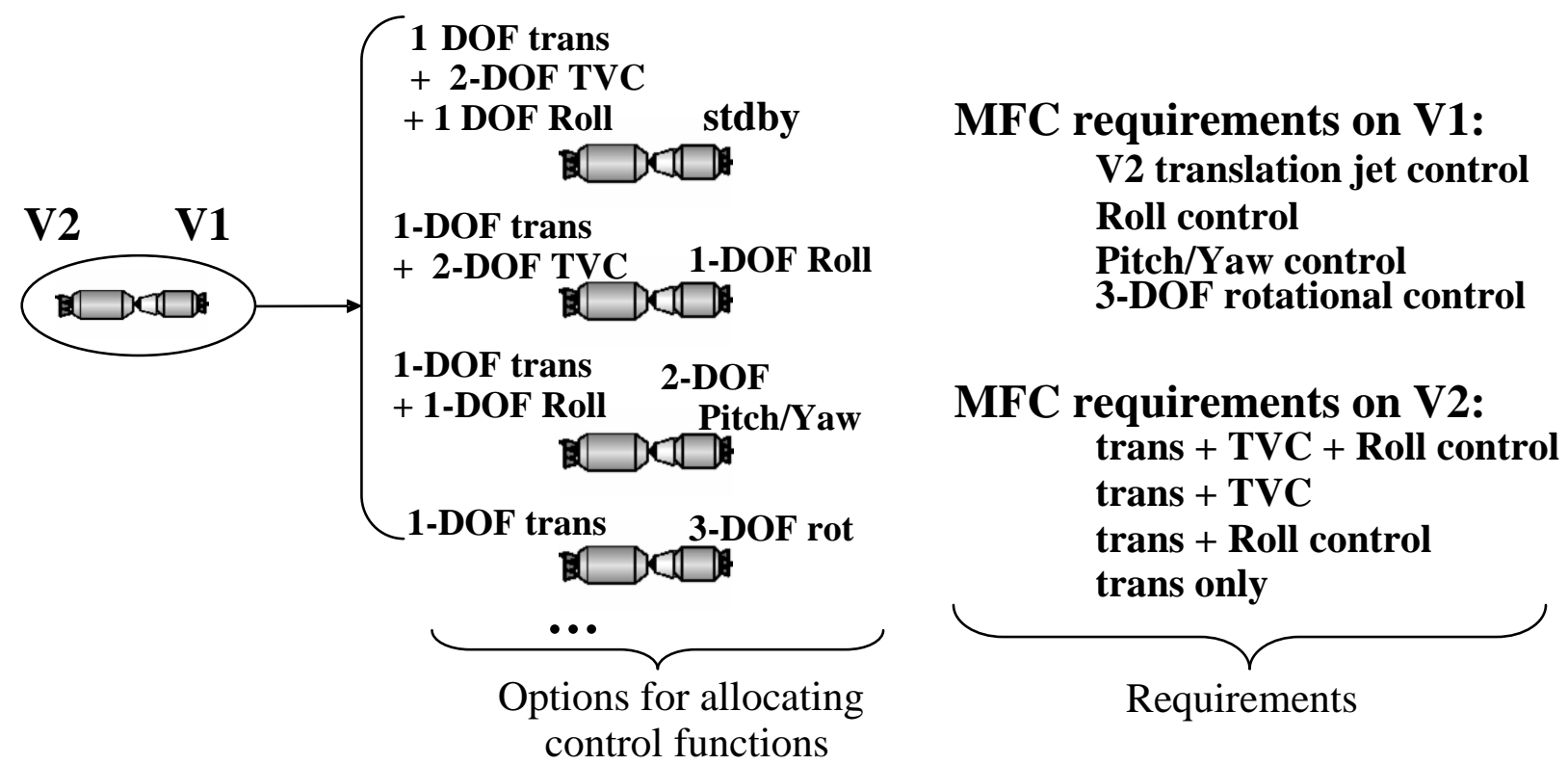

Figure 1 Some options for 4-DOF MFC of two elements.

\subsection{Controllability}

Each element will have independent actuator sets, fully capable of controlling that element, but not necessarily adequate for control of the mated vehicle. Hence, controllability concerns for planned mated configurations must be accounted for as a necessary design requirement. A feasible actuator set must provide sufficient control authority for MFC to meet performance requirements, over all control modes and mated configurations, while operating within allocated budgets including available power, volume, and propellant mass (for example, as required for orbit corrections and attitude control options). A key question that needs to be addressed is which configurations will allow effective MFC. This is basically a control system architecture design issue involving the selection of control type, configuration, and sizing. Key influencing factors that will influence control system architecture selection for controllability include the following:

- Performance requirements, derived from the mission profile

o control accuracy, speed, maneuverability

o disturbance environment (crew motion, fluid motion, docking/undocking loads, payload relocation, gimbal articulation, etc)

- Dynamics of mated vehicles, for all configurations 
- Degree of structural flexibility for all mated configurations

o May require additional sensors and actuators for large flexible structure control

There are many options available in dealing with controllability of a mated vehicle. Many of these options basically involve the selection of control system architectural aspects. The control configuration for MFC can either be localized on one element or distributed over all mated elements. The former option corresponds to Architecture 1 while the latter option corresponds to Architecture 2 and 3 (see Table 1). The trade-offs in terms of for example, simplicity and control effectiveness are described in Table 1.

The option to use a passive stabilization device can also affect controllability of the mated vehicle in terms of S\&C. Gravity gradients could be used to passively stabilize the vehicle in LEO or in lunar orbit, while spin stabilization using momentum bias wheel can be used to design in directional stability of the vehicle at any point in space.

The selection of actuator type and their sizing will also affect the level of controllability for MFC. The options for actuator type include Thrusters, Thrust Vector Control, Reaction Wheels, and CMGs, and a detail and comprehensive discussion of the options are given in [8] and [9]. If an impulsive thrusting RCS gives rise to unacceptable structural flexibility interactions of the mated vehicle, then either a continuously thrusting RCS system or CMG's may be required for controlling the flexural motions in the mated structure. Although defining the necessary functional capabilities of a spacecraft GN\&C system with respect to controllability can be quite obvious, establishing its feasibility or its physical realizability requires a detailed analysis with careful consideration of the various underlying assumptions.

\subsection{Changes in Static and Dynamic Characteristics}

Accurate knowledge of the static and dynamic characteristics of the mated vehicle is crucial for effectively executing GN\&C functions, and at worst dynamic instability can result leading to failures in mission and safety. The problem is that reliable and accurate a priori dynamic models for multiple mated vehicle configurations will be difficult to obtain through analytical modeling even with the aid of ground tests. A reason for this is that the dynamics of a vehicle are expected to change when mated due to inertia and stiffness changes. This is a reflection of the fact that composite vehicle structural modes are not a sum of individual vehicle structural modes. Additional challenges concern the anticipated difficulty in trying to model the stiffness and damping at the mated interface.

Ground testing of various mating configurations will not likely provide adequate data to produce an accurate dynamic model of a mated vehicle. The limiting factors for this includes, (1) multiplicity of configurations in exploration systems architecture that needs to be tested, (2) cost and schedule for highfidelity system level testing in flight configuration, (3) effects of gravity, (4) dynamic changes due to support system and boundary conditions, and (4) scalability of components and joints in dynamic tests. For these reasons, it will be challenging to obtain a close correlation between dynamic test data and control design models that must have sufficiently accurate predictive capability for mated vehicles which will be more complex than any individual element. Ultimately, any successful MFC must perform satisfactorily under configuration changes, parametric variability, increases in dynamical system complexity, increases in the number of state variables, actuators, sensors, disturbance sources, potential coupled failure modes, and finally increases in uncertainties that are difficult to anticipate for the mated system.

Satisfactory MFC requires either high-fidelity models and/or controllers that can robustly adapt on-orbit to discrete configuration changes and system variations, which will likely be dependent on parameter estimation and/or tuning of the control law directly. Relying on accurate high-fidelity models will be of 
lower risk due to its inherent simplicity but will be difficult to impossible if the mated configurations and corresponding variations are too many or not completely known a priori. On the other hand, an on-orbit adaptive control approach is elegant but is difficult to predict off-nominal performance with current state of the art.

A familiar option is to consider a full system dynamic ground testing plan for nominal design and analysis. However, all elements and configurations may not be available for ground test, due to the planned sequence of module development. In addition, larger mated configurations may not be testable in 1-g due to limitations in their structural strength.

At present, on-orbit system testing and adaptive/reconfigurable control appear necessary to enhance the robustness of MFC. There are different aspects of the robustness problem that we can address. First, one could try to develop mathematical and simulation models of all planned mated configurations that will accurately match actual operating environments. While techniques to develop mathematical and simulation models for MFC are well established, ensuring that these models will accurately match actual operating environments is difficult. Ensuring this will require a high level of success in V\&V of MFC system performance. The second aspect is to try to envelope uncertainties in the model and disturbances for the mated vehicle in terms of constraints and parameters, for robust control analysis and design. This envelope, if physically justified and validated, will ensure that the methods for robust control analysis and design will be effective. The third aspect is to consider different types of control structures for coping with widely varying system configurations and system uncertainties.

Three levels of on-orbit system testing and adaptive control/controller sophistication are envisioned for MFC.

\section{$\underline{\text { Standard Approach }}$}

This involves closed-loop on-orbit dynamic testing and system identification aimed at refining (hence previous analytical modeling and ground tests should be helpful) the plant model of a mated vehicle for MFC application. Following a successful refining or update of the plant model, controller parameters (such as filter gains, band-pass, notch frequencies, etc) can be updated based on appropriate design algorithms. Parameters are updated manually. Nominally, controllers will be gain scheduled.

\section{Indirect adaptive control}

This approach is similar to the "Standard" approach but the controller parameter updates, based on a controller re-design algorithm, are done autonomously. Although the assumption of autonomy is elegant and makes the adaptation process convenient, $\mathrm{V} \& \mathrm{~V}$ is difficult because it is difficult to simulate realistic environments, especially unanticipated off-nominal conditions, with confidence.

\section{Direct adaptive control}

This option is similar to the indirect adaptive approach except that the controller parameters are updated directly without a need for plant model refinement or a controller re-design algorithm. Additional functional capability can include, for example, mass properties identification, sensor and actuator FDIR, and fault recovery strategy. However, as in any autonomous and adaptive control system, $\mathrm{V} \& \mathrm{~V}$ will be a challenge.

In any approach envisioned, analytical modeling with V\&V through ground testing and limited flight tests will definitely be valuable to a successful on-orbit system testing and adaptive/reconfigurable control approach. Evaluating the impact of structural flexibility in MFC will require a more detailed dynamic model beyond 6-DOF such as a finite-element model. Additional sensors may be needed in-situ to measure flexure and, if needed, as part of a system to actively suppress it, for example using "smart structures" technology. 


\subsection{Attitude Stability \& Control of Mated System}

A major goal of any MFC system is to provide GN\&C capability. First, we need to clarify what is unique to GN\&C capability of a MFC as related to each spacecraft element. For the Guidance and Navigation part, we note that both functionally and from a performance perspective, the required sensing, computing, commanding and controlling position and velocity, to track a desired trajectory is similar to each unmated vehicle. On the other hand, for attitude S\&C functions that involve the sensing and controlling vehicle attitude and attitude rates and maintain attitude stability throughout mission, the performance will strongly depend on mated system details (for example, element inertias, structural joint, actuators, control algorithms, control structure, etc.). Consequently, we examine how attitude S\&C options can influence MFC architecture options.

Figure 2 is a schematic of an interconnection of a 2-element mated vehicle configuration with a common MFC law. Again, V1 and V2 denote vehicle 1 and vehicle 2, respectively.

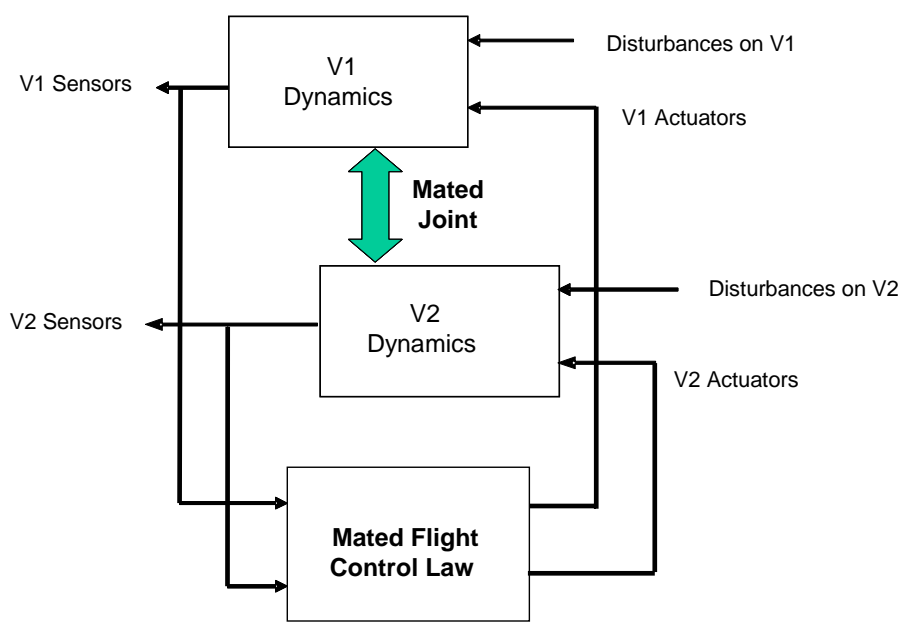

Figure 2 Schematic of 2-element mated system interconnected with a mated flight control law

The MFC performance concerning attitude S\&C will depend on many factors including the following: (i) combined disturbances, (ii) combined mass properties (Inertia, CG, etc.), (iii) mated joint and structural flexibility change, (iv) combined actuators and sensors, and (v) mated flight control law and structure. Notice from the above list that the first three factors are given (defined by mission, payload, operational requirements, structural, etc.) whereas the latter two can be considered MFC architecture options and may be chosen. We consider these latter factors next in more detail.

The control architecture for MFC will require the specification of sensors, actuators, and control law as illustrated in Figure 3 where the quantities (C11, C12, C21, C22) denote control gains or more generally operators acting on sensor variables.

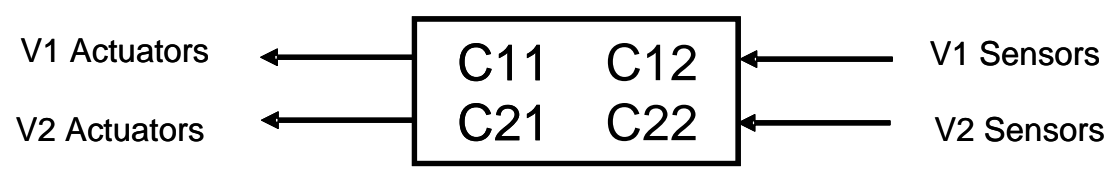

Figure 3 Schematic of Mated Flight Control Law 


$$
\text { or }\left\{\begin{array}{l}
u_{1} \\
u_{2}
\end{array}\right\}=\left[\begin{array}{ll}
C_{11} & C_{12} \\
C_{21} & C_{22}
\end{array}\right]\left\{\begin{array}{l}
y_{1} \\
y_{2}
\end{array}\right\} \ldots \text { and more generally, }\left\{\begin{array}{l}
u_{1} \\
u_{2}
\end{array}\right\}=\left\{\begin{array}{l}
f_{1} \text { (states, parameters,...) } \\
f_{2} \text { (states, parameters,...) }
\end{array}\right\}
$$

In addition, depending on the MFC structure, the control law can be fully coupled across all sensors to actuators or highly structured as in for example, a spatially decentralized control architecture with local feedback. What combinations of actuators and sensors selected, including mated flight control law and its structure can be viewed as quantities chosen in (C11, C12, C21, C22). Figure 4 shows all possible combinations of sensors and actuators for 2-element case. Infeasible combinations are denoted by an asterisk.

\begin{tabular}{|c|c|c|c|c|}
\hline & None & $\begin{array}{c}\text { V2 } \\
\text { Sensors }\end{array}$ & $\begin{array}{c}\text { V2 } \\
\text { Actuators }\end{array}$ & $\begin{array}{c}\text { V2 } \\
\text { Sen \& Act }\end{array}$ \\
\hline None & $*$ & $*$ & $*$ & {$\left[\begin{array}{cc}0 & 0 \\
0 & C_{22}\end{array}\right]$} \\
\hline $\begin{array}{c}\text { V1 } \\
\text { Sensors }\end{array}$ & $*$ & $*$ & {$\left[\begin{array}{cc}0 & 0 \\
C_{21} & 0\end{array}\right]$} & {$\left[\begin{array}{cc}0 & 0 \\
C_{21} & C_{22}\end{array}\right]$} \\
\hline $\begin{array}{c}\text { V1 } \\
\text { Actuators }\end{array}$ & $*$ & {$\left[\begin{array}{cc}0 & C_{12} \\
0 & 0\end{array}\right]$} & $*$ & {$\left[\begin{array}{ll}0 & C_{12} \\
0 & C_{22}\end{array}\right]$} \\
\hline $\begin{array}{c}\text { V1 } \\
\text { Sen \& Act }\end{array}$ & {$\left[\begin{array}{cc}C_{11} & 0 \\
0 & 0\end{array}\right]$} & {$\left[\begin{array}{cc}C_{11} & C_{12} \\
0 & 0\end{array}\right]$} & {$\left[\begin{array}{ll}C_{11} & 0 \\
C_{21} & 0\end{array}\right]$} & {$\left[\begin{array}{ll}C_{11} & C_{12} \\
C_{21} & C_{22}\end{array}\right]$} \\
\hline
\end{tabular}

Figure 4 Feasible sensor and actuator combinations for 2-element MFC

The question arises as to what will be the most effective (or performance enhancing) framework for control design involving actuators, sensors, and controller structure, i.e., MFCLS. The first step is to find all MFCLS configurations that are "feasible", i.e., stabilizable.

Notice that for 2-element case, the three classes of control law structure, earlier referred to as Architecture 1, 2, and 3 (see Table 1) corresponds to special cases given in Figure 4, i.e., Architecture 1 corresponds to $\left[\begin{array}{cc}C_{11} & 0 \\ 0 & 0\end{array}\right],\left[\begin{array}{cc}0 & 0 \\ 0 & C_{22}\end{array}\right]$, while Architectures 2 and 3 correspond to $\left[\begin{array}{ll}C_{11} & C_{12} \\ C_{21} & C_{22}\end{array}\right]$. The other architectures such as $\left[\begin{array}{cc}C_{11} & C_{12} \\ 0 & 0\end{array}\right],\left[\begin{array}{ll}C_{11} & 0 \\ C_{21} & 0\end{array}\right],\left[\begin{array}{cc}0 & 0 \\ C_{21} & C_{22}\end{array}\right],\left[\begin{array}{ll}0 & C_{12} \\ 0 & C_{22}\end{array}\right]$ correspond to architectures with redundancies while the remaining architectures $\left[\begin{array}{cc}0 & C_{12} \\ 0 & 0\end{array}\right],\left[\begin{array}{cc}0 & 0 \\ C_{21} & 0\end{array}\right]$ correspond to the case with split actuators/sensors, i.e., actuators from one element and sensors from another element. 


\subsection{CONCLUDING REMARKS}

Several unique issues related to MFC have been broadly identified in this study. Among these, the issue involving MFC of two vehicles under variable static and dynamic characteristics have been highlighted in this paper. This brief study on MFC has provided a good starting point for more detailed analysis. However, other more basic considerations such as building-in sufficient redundancies of MFC against failures or providing sufficient control authority, so as to satisfy GN\&C performance requirements for all control modes while fitting within the allocated mass, power, and volume budgets, have not been addressed adequately, even at a cursory level. This will require a much more detailed analysis based on performance requirements of mated systems and feasible configurations and constraints on each element.

Given the inherent complexity in mated vehicle systems and the history of system failures, special attention must be given during the selection of candidate MFC architectures to issues involving offnominal operations and performance. Architecture 2 appears most suitable for MFC for the following reasons: (i) it provides command capabilities to all available GN\&C resources, (ii) it provides sufficient redundancies in actuators and sensors to accommodate failures, (iii) it is more effective in producing control torques, (iv) and is more suited for controller reconfigurations. Architecture 3 is the most advanced but may not be technologically mature. All architectures will require some level of robustness and intelligent/adaptive and reconfigurable control capabilities due to unanticipated and anticipated system changes such as, mass property changes (with mating, propellant depletion, payload shifts, etc), increase in vehicle flexure with mating, mating transients (contact \& controller induced), integrated GN\&C failure modes, and separation and abort loads. Given the need to incorporate MFC system that encompasses reconfigurability, adaptiveness, and robustness, feasible V\&V plans to assure MFC performance will be critical for success.

\section{ACKNOWLEDGEMENTS}

The authors would like to thank their colleagues Suresh Joshi, Sean Kenny, Louis Nguyen, and John Ruppert of NASA and Tom Cochrane of Raytheon who participated in an earlier study on mated flight control.

\section{REFERENCES}

[1] Exploration Crew Transportation System Requirements Document (Spiral 2), ESMD-RQ-0012 Preliminary (Rev D), February 22, 2005, NASA Headquarters, Washington DC. www.exploration.nasa.gov.

[2] Exploration Crew Transportation System Requirements Document (Spiral 3), ESMD-RQ-0013 Preliminary (Rev D), February 22, 2005, NASA Headquarters, Washington DC. www.exploration.nasa.gov.

[3] "International Space Station (ISS) to Orbital Space Plane (OSP) Interface Requirements Document”, International Space Station Program, Draft 1, June 10, 2003, NASA JSC.

[4] Orbital Space Plane (OSP) Level 2 System Requirements Document (SRD), MSFC-RQMT-3360 Baseline, September 5, 2003, MSFC.

[5] Apollo Experience Report - Guidance and Control Systems, Digital Autopilot Design Development, W. H. Peters, and K.J. Cox, NASA TN D-7289, Washington DC, June 1973. 
[6] “Apollo Guidance and Navigation”, Space Navigation, Guidance, and Control, Vol. 1 of 2, June 1965, MIT Instrumentation Laboratory, Cambridge, MA, Chapter II-2.

[7] Interface Definition Document (IDD) for International Space Station (ISS) visiting vehicles (VVs), SSP 50235, International Space Station Program Office, February 10, 2000, NASA JSC.

[8] Space Mission Analysis and Design, J.R. Wertz, and W.J. Larson (editors), Kluwer Academic Publishers, Norwell, MA, 1991, Section 11.1.

[9] Human Spaceflight: Mission Analysis and Design, W.J. Larson, and L.K. Pranke (editors), McGraw Hill Inc., New York, 1999, Chapter 19

\section{GLOSSARY OF ACRONYMS}

CCI - Command and Control Intercommunication

CMG - Control Moment Gyroscopes

C\&DH - Command and Data Handling

CSM-LM - Command Service Module-Lunar Module (Apollo)

C2 - Command \& Control

DOF - Degree of Freedom

FC - Flight Control

FDIR - Failure Detection, Isolation and Recovery

GN\&C - Guidance, Navigation, \& Control

ISS - International Space Station

MFC - Mated Flight Control

MFCLS - Mated Flight Control Law Structure

OSP - Orbital Space Plane

RCS - Reaction Control System

S\&C - Stability and Control

TVC - Thrust Vector Control

V1,V2 - Vehicle 1, Vehicle 2

VV - Visiting Vehicle

V\&V - Validation and Verification 